\title{
Compton Scattering in Electron Excited Energy Dispersive X-ray Spectra
}

\author{
Nicholas W. M. Ritchie*, Dale E. Newbury* and Abigail P Lindstrom* \\ * Surface and Microanalysis Science Division, National Institute of Standards and Technology, \\ Gaithersburg, MD 20899-8372
}

Trace element analysis using electron-excited energy dispersive x-ray spectrometry is always a challenge. Unless one is careful it is easy to mistake coincidence peaks, escape peaks, absorption edges and other artifacts for trace quantities of non-existent elements. When studying energetic $\mathrm{x}$ rays from particulate samples there is an additional artifact which we believe we have identified and explained for the first time - Compton scattering. Compton scattering is a process which is familiar to practitioners of x-ray fluorescence spectrometry (XRF). In the Compton process, an x-ray loses energy when it scatters from an electron. XRF spectra often show a background resulting from Compton scattering of the incident $\mathrm{x}$-ray flux. In a XRF spectrum collected with a rhodium source, it is common to see peaks resulting from the $20.2 \mathrm{keV} \mathrm{Rh} \mathrm{K} \alpha$ characteristic lines but shifted by approximately a kilo-electron volt lower in energy. The magnitude of the Compton shift depends upon the x-ray energy and the scatter angle but increases with $\mathrm{x}$-ray energy and can be $0.7 \mathrm{keV}$ at $13.6 \mathrm{keV}[1]$. However, Compton scattering is not typically seen in electron excited energy dispersive spectra. We have discovered that under certain conditions it is possible to see a signal due to Compton scattering. Furthermore, we can predict the approximate magnitude and shape of the signal as a function of particle and substrate composition.

The key to understanding why we typically don't see Compton scattering and yet why under certain circumstances it is possible is the relative cross sections for the photo-absorption and Compton processes [2]. The cross section is proportional to the likelihood of certain process occurring as an x-ray traverses a distance in a material. If the photo-absorption cross section is much larger than the Compton cross section (as is typically the case) then almost all x-rays will be absorbed before they Compton scatter. Once absorbed the x-ray is essentially lost. Even when an xray does Compton scatter, the scattered x-ray is likely to be absorbed before it can reach the detector. Thus to observe Compton scattering we need a material in which the Compton cross section is of similar magnitude to the photo-absorption cross section. Higher $\mathrm{Z}$ materials (such as those which produce characteristic $x$-rays above $10 \mathrm{keV}$ ) tend to have larger photo-absorption cross sections but relatively small Compton cross sections. In general, materials which produce energetic x-rays are not suitable for observing Compton scattering. Neither are low Z materials suitable because they don't produce sufficiently energetic x-rays. However low $Z$ materials do have a more favorable relationship between Compton scattering and photo-absorption. To observe Compton scattering we need a low $\mathrm{Z}$ material to produce the scatter and a high $\mathrm{Z}$ material to produce the $\mathrm{x}$-rays. We can engineer this situation (or mistakenly fall into it) by placing a high $\mathrm{Z}$ particle on a low $\mathrm{Z}$ substrate. For example, a $\mathrm{U}_{3} \mathrm{O}_{8}$ (see FIG 1.) or a $\mathrm{SrF}_{2}$ particle on a $\mathrm{C}$ substrate satisfy both requirements. In fact, the Compton peak from the uranium L line looks similar to a L line from trace Th. Since these elements are often found together it is easy to imagine a circumstance in which Compton scatter from the $\mathrm{U} \mathrm{L}$ line is mistaken for Th at approximately the 0.8 weight percent level.

Fortunately, there are simple ways to avoid this artifact. One is to mount the particle on a higher $\mathrm{Z}$ substrate like Al or Si. Alternatively mount the particle on a thin film like a TEM grid or Mylar. Since the cross section for Compton is small, it takes a bulk substrate to produce sufficient scatter to observe. 
[1] O. Klein \& Y. Nishina, Nature 122 (1928) 398-399

[2] W.H. McMaster N.K. Del Grande, J.H. Mallett and J.H. Hubbell, Lawrence Livermore National Laboratory Report UCRL-50174 (section I 1970, section II 1969, section III 1969 and section IV 1969).

[3] E. L. Garner, L. A. Machlan \& W. R. Shields, NBS Special Pub. 260-27 (1971)

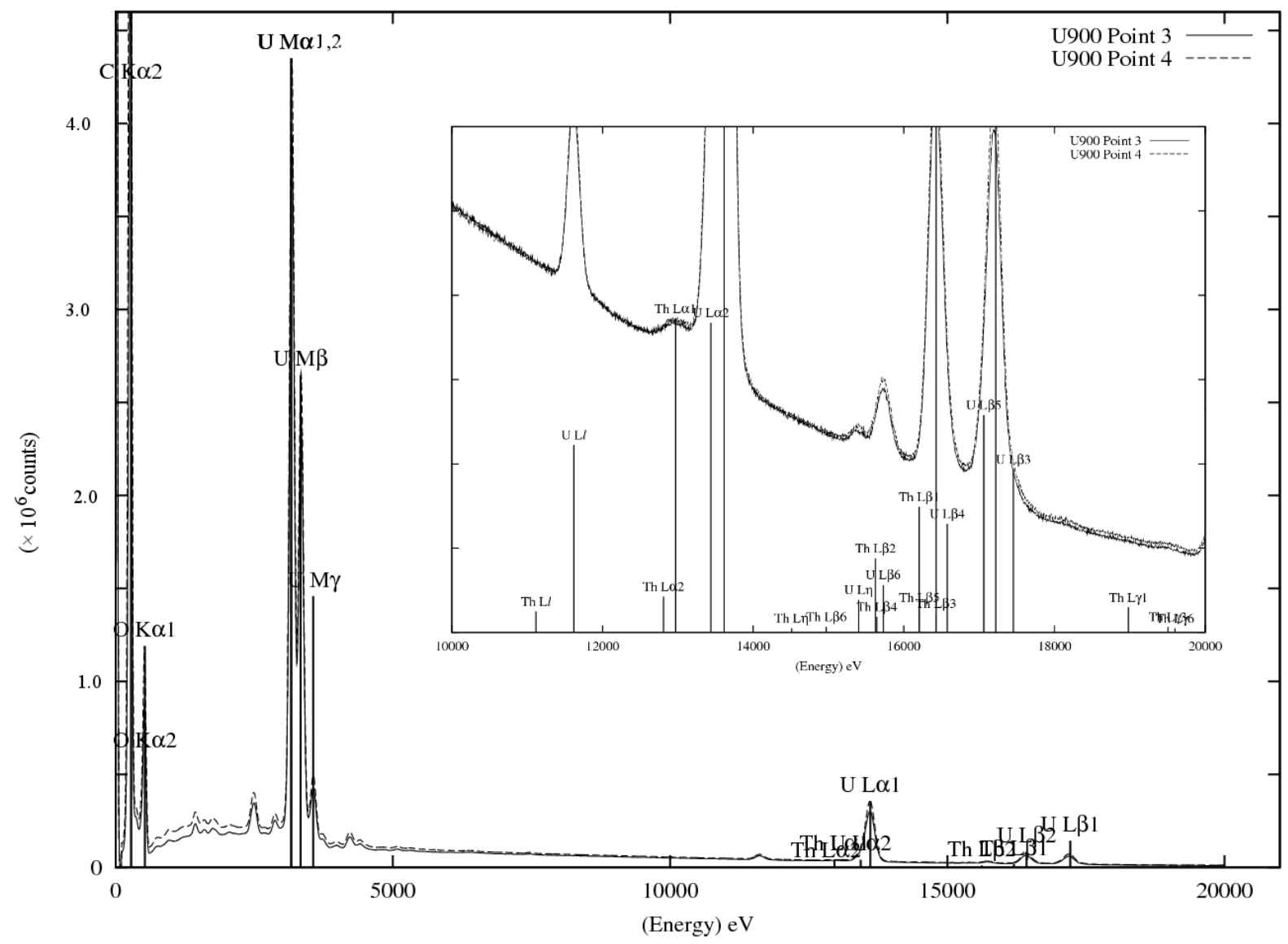

FIG 1: Spectra from two high purity uranium oxide particles (U900[3]). The spectra show a feature incorrectly labeled as Th $L \alpha$ on the low energy side of the $U \mathrm{~L} \alpha$ line. We attribute the artifact to Compton scattering of the $\mathrm{U} L \alpha$ by the bulk carbon substrate on which the particle rested. 